\title{
Uma Proposta para Melhoria do Planejamento e Controle da Produção em Projetos de Construção Civil
}

\author{
Renato Alexandre C. Freitas ${ }^{1}$, Reinaldo B. Braga ${ }^{1}$, Carina T. de Oliveira ${ }^{1}$ \\ ${ }^{1}$ Laboratório de Redes de Computadores e Sistemas (LAR) \\ Instituto Federal de Educação, Ciência e Tecnologia do Ceará (IFCE)
}

\begin{abstract}
The Production Planning and Control (PCP) in the construction industry has advanced with the use of Information and Communication Technologies (ICT). However, some challenges are still open in this area, such as how to overcome schedule delays. This article presents a proposal to improve the PCP in civil construction projects, applying a lean methodology combined with a mathematical model to present important performance indicators for decision making. The proposal was modeled and implemented as a new module in a real construction web system. Finally, a case study is presented to demonstrate the feasibility of applying the proposal in a real civil construction project.
\end{abstract}

Resumo. O Planejamento e Controle da Produção (PCP) na indústria da construção civil têm avançado com o uso das Tecnologias da Informação $e$ Comunicação (TIC). Porém, alguns desafios ainda existem nessa área, tal como superar atrasos nos cronogramas. Este artigo apresenta uma proposta para aprimoramento do PCP em projetos de construção civil, sendo aplicada uma metodologia enxuta aliada a um modelo matemático para apresentar indicadores de desempenho importantes para a tomada de decisão. A proposta foi modelada e implementada como um novo módulo em um sistema web real de construção civil. Por fim, é apresentado um estudo de caso para demonstrar a viabilidade de aplicação da proposta em um projeto real de construção civil.

\section{Introdução}

No Brasil, a Indústria da Construção (IC) tem uma representatividade significativa no Produto Interno Bruto (PIB) nacional. Em 2018, o setor gerou 278 bilhões de reais em valor de incorporações, obras e/ou serviços da construção. Nesse cenário, destaca-se a participação do setor público como um importante cliente que, em 2009, representava 43,2\%, já em 2018 passou a ser 30,7\% [IBGE 2019].

Apesar da incontestável relevância da IC para a movimentação da economia brasileira, o setor ainda enfrenta inúmeros desafios quando as temáticas são planejamento e gerenciamento de prazos. Em 2019, o Tribunal de Contas da União (TCU), após auditoria em 38 mil contratos referentes a obras públicas no Brasil, apontou que cerca de $37 \%$ das obras encontravam-se paralisadas, contabilizando um total de cerca de 14 mil obras.

Diante de tal problemática, cabe a realização de estudos direcionados às boas práticas de gestão de cronogramas associadas ao uso de Tecnologias da Informação e Comunicação (TICs), capazes de apoiar no planejamento dos cronogramas e predição de atrasos nas atividades planejadas de obras da IC. 
Como contribuição nesse sentido, este trabalho apresenta uma proposta para melhoria do Planejamento e Controle da Produção (PCP) em projetos de construção civil. O processo de construção da proposta é apresentado em cinco etapas principais, sendo, ao final, mostrado que a proposta foi implementada como um módulo web em um sistema real de gestão para construção civil que permite controlar as atividades planejadas a partir dos princípios da metodologia Last Planner System (LPS). Por fim, é apresentado um estudo de caso para demonstrar a viabilidade da proposta em um projeto real da IC.

\section{Trabalhos Relacionados}

No trabalho de [Moura and Formoso 2009] é realizado um estudo cujo objetivo foi o desenvolvimento e implantação de um sistema de indicadores para realização de benchmarking na IC. A partir de dados de empresas participantes do projeto, foi realizada uma análise quantitativa de indicadores de PCP para identificar os impactos positivos e negativos da adoção do LPS em empreendimentos da construção civil com ênfase no custo e no prazo. O estudo foi realizado a partir de dados reais de projetos já realizados.

Já no estudo de [Silva et al. 2019] é destaque o uso da Building Information Modeling (BIM). Mais detalhadamente, o trabalho realiza uma análise para identificar e classificar os benefícios e dificuldades envolvidas na associação da BIM ao PCP. Apesar de observadas melhorias no PCP com a adoção da BIM, os autores destacam que seu processo de implementação é árduo dado o intenso trabalho de modelagem 3D e consequente integração disciplinada com o cronograma da obra. Em relação ao PCP, o controle, a representação e a distribuição de informações referentes ao cronograma tendem a apresentar inconsistências, sendo que, na maioria dos casos, este é um processo manual e, principalmente, pode não ser realizado de forma apropriada.

\section{Proposta}

Inicialmente, é importante destacar que a proposta deste artigo foi toda desenvolvida a partir de uma demanda de mercado oriunda de um projeto de Pesquisa, Desenvolvimento e Inovação (PD\&I) financiado pela EMBRAPII ${ }^{1}$, tendo como demandante a empresa Quanta Consultoria ${ }^{2}$, uma empresa com forte atuação a nível nacional e internacional que opera nas áreas de consultoria, engenharia e planejamento urbano. Apesar da demanda ser proveniente de uma empresa específica, a proposta apresentada nesta seção pode ser aplicada/replicada por outras empresas, governos e/ou sistemas.

Assim como apontado pela empresa parceira do projeto e pela literatura [Abou Ibrahim et al. 2019] [Lucko et al. 2019], há duas barreiras principais que dificultam a capacidade dos planejadores na concordância entre a carga de trabalho dos operários e sua capacidade no planejamento de curto prazo de uma obra de construção civil: (i) a incapacidade de prever a carga de trabalho que pode ser absorvida pelos trabalhadores da obra e (ii) a quantidade de atividades que estarão prontas com antecedência.

Neste trabalho, a superação dessas barreiras consiste na proposta de melhoria do Planejamento e Controle da Produção (PCP). A proposta é apresentada em cinco etapas principais, que são detalhadas na sequência dessa seção. Ao final, a proposta permite que

\footnotetext{
${ }^{1}$ Empresa Brasileira de Pesquisa e Inovação Industrial - https://embrapii.org.br/

${ }^{2}$ https://www.quantaconsultoria.com/
} 
gestores de obras de construção civil visualizem em uma interface web amigável uma projeção de atrasos e indicadores de desempenho.

\subsection{Etapa 1 - Análise de cronogramas físico-financeiro}

A primeira etapa da proposta passa pela análise de planilhas fornecidas pela empresa parceira, dentre elas destacam-se: o Plano Contratual (um plano macro do projeto que contempla todas as atividades desde a mobilização de equipamentos e pessoal até a conclusão de uma obra, bem como suas respectivas quantidades e valores); o cronograma físico-financeiro (que representa a distribuição do que foi planejado e do avanço físico real em percentual e a relação com seus respectivos custos); o boletim de medição (um documento utilizado para verificar a compatibilidade entre o que foi executado e o que está previsto no projeto e nos quantitativos do orçamento); e a Curva $S$ (que também é uma representação gráfica que permite observar os desvios daquilo que foi planejado em comparação com o realizado). O material fornecido foi utilizado para o entendimento dos processos envolvidos na gestão de uma obra e, principalmente, para nortear o desenvolvimento de um módulo em um sistema web, objeto final deste estudo.

Apesar de sua importância, constatamos que guiar-se apenas pelo cronograma físico-financeiro pode não garantir o PCP e, principalmente, evitar a problemática causada pelos atrasos. Em particular, com base nas observações pontuadas, talvez não seja possível garantir um comprometimento no que diz respeito ao planejado e executado. Evidenciou-se que existem desvios entre os pacotes de trabalho planejados e realizados. Concluiu-se que se fazia necessário implementar uma solução complementar que aproximasse os valores medidos dos planejados no cronograma físico-financeiro.

A partir do que foi discutido na Etapa 1, surgiram os seguintes questionamentos principais a serem respondidos na próxima etapa: qual metodologia é capaz de aprimorar os resultados apresentados em um cronograma físico-financeiro? Como um sistema web pode ser utilizado para permitir o acompanhamento da execução da obra pelos gestores?

\subsection{Etapa 2 - Análise de práticas modernas de gestão em projetos de construção}

$\mathrm{Na}$ Etapa 2, realizou-se um levantamento bibliográfico exaustivo de práticas modernas de gestão em projetos de construção. Nesse contexto, percebeu-se a ampla adoção da filosofia da construção enxuta (Lean Construction) [Mubarak 2015], que tem sido largamente recomendada por especialistas e instituições como a Lean Construction Institute $(\mathrm{LCI})^{3}$. Na filosofia lean, existem várias ferramentas com o propósito comum de melhoria contínua. Uma delas é o Last Planner System (LPS), que tem como propósito reduzir as camadas hierárquicas no gerenciamento da construção e estendê-la aos atores baseados em campo, capacitando-os no processo de gestão da construção para otimizar a alocação dos recursos, planejamento semanal, cronogramas e execução do trabalho. O principal indicador utilizado em LPS é a Porcentagem Planejada Concluída (PPC), que é calculado ao término da semana a partir das tarefas completas.

O LPS é organizado em níveis hierárquicos: planejamento mestre ou de longo prazo (master plan), planejamento de médio prazo (lookahead planning) e planejamento de comprometimento ou de curto prazo (weekly work planning). Os objetivos globais e restrições que governam o projeto como um todo são estabelecidos pelo master plan. Já

\footnotetext{
${ }^{3}$ https://www.leanconstruction.org/
} 
o planejamento lookahead, por sua vez, tem como principal função dar forma e controlar o fluxo de trabalho e, por fim, o weekly work planning tem como papel principal avaliar o que pode e o que deve ser feito baseado nos recursos disponíveis e no cumprimento de pré-requisitos a fim de atribuir corretamente os pacotes de trabalho às equipes e gerenciar os compromissos com as mesmas [Ballard 2000].

Apesar das melhorias oportunizadas pela LPS, o uso inadequado das técnicas de planejamento e controle recomendadas podem apresentar desvios e produzir métricas e indicadores de desempenho com resultados contrários à realidade. De acordo com [Ballard 2000], o PPC apresenta algumas limitações, por exemplo: é possível aumentar artificialmente este indicador através do estabelecimento de metas semanais inferiores à capacidade de produção das equipes, ou dividir uma tarefa em pequenos pacotes de trabalho. Isso pode resultar em um PPC com valor maior em comparação aos pacotes de trabalho bem definidos.

Com base nos princípios de definição de LPS, pode-se destacar as seguintes hipóteses: (i) mesmo não sendo necessário o uso de software para a adoção de LPS, uma ferramenta web pode auxiliar na distribuição de informações com todos os stackholders em tempo hábil; (ii) métodos matemáticos podem potencializar a LPS através de projeções do weekley work planning; (iii) é possível formar uma base de conhecimento a partir dos indicadores de LPS.

\subsection{Etapa 3 - Análise de modelos matemáticos}

Nesta etapa do processo, a pesquisa bibliográfica foi direcionada com base na hipótese apresentada na etapa anterior, que propõe a possibilidade do uso de modelos matemáticos para antecipar o PPC através de projeções antes do término do weekly work planning. Assim, constatou-se a viabilidade do uso de Funções de Singularidade na predição de atrasos em pacotes de trabalhos gerenciados através da LPS [Lucko et al. 2019]. Mais especificamente, para esta proposta, buscou-se identificar, a partir da taxa de produção, variações no tempo de execução dos pacotes de trabalho que possam resultar em atrasos. A representação dessas funções pode ser observada na Equação 1.

$$
\langle x-a\rangle^{n}=\left\{\begin{array}{ccc}
0 & \text { para } & x<a, \\
(x-a)^{n} & \text { para } & x \geq a,
\end{array}\right.
$$

sendo $x$ a variável em consideração e $a$ a representação do ponto de ativação. O expoente $n$ indica o comportamento no gráfico. Se $n=0$, tem-se uma etapa "estacionária". Caso $n=1$, o resultado será uma inclinação.

De acordo com [Lucko et al. 2019], a função é executada em um loop utilizandose a Taxa de Produção Atual e a Taxa de Produção Esperada até que se alcance $100 \%$ das atividades planejadas, conforme destacado nas Equações 2 e 3, respectivamente.

$$
\begin{gathered}
W n(t)=0 \cdot\langle t-0\rangle^{0}+0.5 \cdot\langle t-0\rangle^{1}-0.5 \cdot\langle t-8\rangle^{1}-4 \cdot\langle t-8\rangle^{0} \\
W n(t)=0 \cdot\langle t-0\rangle^{0}+0.5 \cdot\langle t-0\rangle^{1}+1 \cdot\langle t-2\rangle^{1}-3 / 2 \cdot\langle t-4\rangle^{1}-4 \cdot\langle t-4\rangle^{0}
\end{gathered}
$$

Após a execução das equações, os dados resultantes são utilizados para a projeção em um gráfico, como será mostrado na Seção 5. 


\subsection{Etapa 4 - Modelagem da proposta em um sistema de gestão de construção civil}

A Etapa 4 consistiu na modelagem dos conceitos e decisões descritos nas etapas anteriores para posterior prova de conceito em um sistema real de gestão de construção civil. O sistema escolhido para prova de conceito foi o Bentham Obras, um sistema de gestão inteligente para a construção civil [Batista et al. 2019]. O desenvolvimento do Bentham Obras foi fomentado por meio de uma parceria com a EMBRAPII. A solução foi desenvolvida pelo Laboratório de Redes de Computadores e Sistemas (LAR)/IFCE a partir da demanda da Quanta Consultoria, citada anteriormente.

O sistema é dividido em dois módulos: uma versão web voltada para utilização em escritório e um aplicativo mobile para utilização em campo, como, por exemplo, no canteiro de obras. Apesar do alto grau de inovação tecnológica do Bentham Obras, antes deste trabalho, o sistema não disponibilizava funcionalidades para o nível de PCP, conceito que vem sendo discutido desde o início desse artigo.

Assim, durante a Etapa 4, realizou-se um estudo aprofundado das tecnologias, arquitetura e do banco de dados do Bentham Obras. De modo geral, as seguintes aspectos foram identificados para a fase de modelagem: $(i)$ a entidade que antes controlava e concentrava todas as atividades de uma obra tornou-se responsável pelo master plan; (ii) a hierarquia é complementada com a estrutura da look ahead planning, responsável pela documentação das atividades, equipes e suas respectivas necessidades; (iii) finalmente, foi definida a estrutura para a weekly work planning. Neste nível, as atividades priorizadas e sem impedimentos são documentadas.

\subsection{Etapa 5 - Implementação da proposta em um sistema de gestão de construção civil}

Nesta etapa, os recursos referentes ao lookahead planning e weekly work planning foram implementados e integrados a API do sistema Bentham Obras. No sistema, o lookahead planning permite selecionar as atividades do master plan, documentar as necessidades de cada pacote de trabalho, bem como a equipe responsável. Após o planejamento, o usuário responsável replica o plano semanal na tela weekly work planning, indicando o comprometimento de cada equipe. Este recurso serve principalmente para comunicar aos gestores o andamento das atividades semanais. Diariamente, registra-se as medições referentes às atividades executadas e se foram completadas ou não. Com este registro é possível calcular: a Taxa de Produção Esperada, a Taxa de Produção Atual, a quantidade de dias de atraso, o PPC e a melhoria necessária na taxa de produção.

\section{Estudo de Caso}

Nesta seção, um estudo de caso é apresentado para demonstrar a viabilidade e os benefícios da aplicação da proposta detalhada na Seção 3 em um projeto real de construção civil. O estudo de caso escolhido é no cenário de construção de um reservatório de água, denominado para este estudo de "Reservatório ABC".

A Figura 1 ilustra a tela para visualização do master plan do projeto em questão. Na nomenclatura do sistema web Bentham Obras, o recurso disponível para controle de tarefas é denominado "Produto", que é a representação de uma parte em um contrato de construção. Portanto, neste estudo de caso, deve-se considerar um "Produto" como o master plan da LPS. 


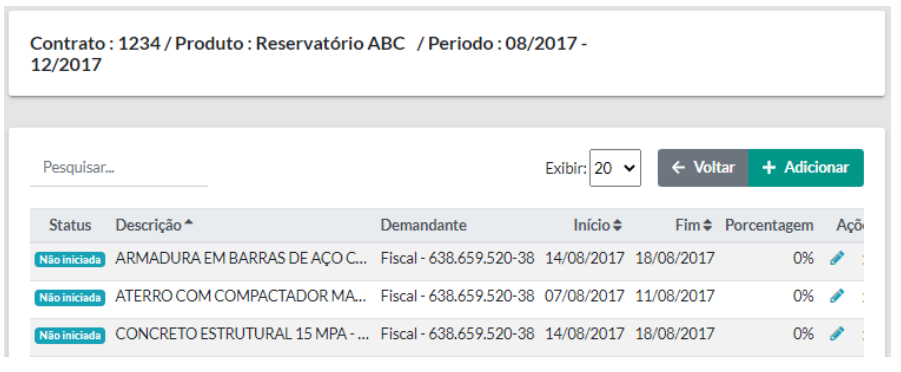

Figura 1. Exemplo de tela de um Produto (master plan).

A partir de um master plan, as tarefas podem ser selecionadas para compor o lookahead planning, que no sistema recebe a nomenclatura de Planejamento de Médio Prazo (Figura 2). Mais especificamente, através dessa nova tela/funcionalidade disponibilizada neste trabalho, os líderes de equipe que estão em campo (chamados no LPS de "últimos planejadores"; do inglês, last planners) selecionam apenas as tarefas para compor o plano de médio prazo, conforme definido na LPS.

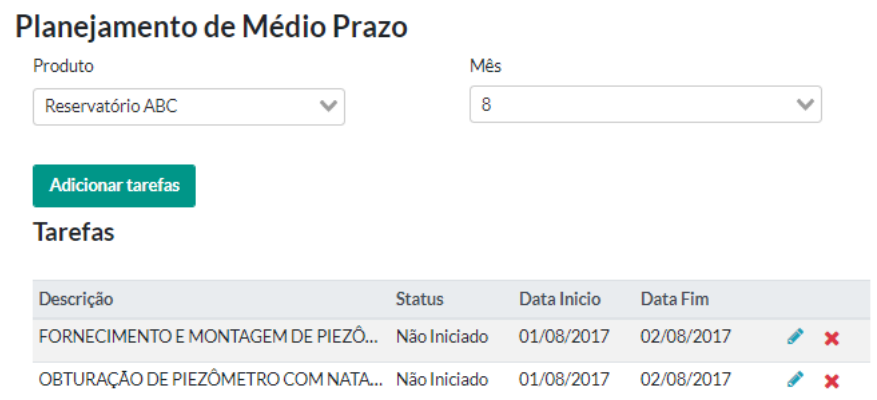

Figura 2. Exemplo de tela para composição do Planejamento de Médio Prazo (lookahead planning).

Seguindo para o próximo nível da LPS, as tarefas agrupadas no lookahead planning podem, por sua vez, também serem agrupadas em um weekly work planning, denominado no sistema de Planejamento de Curto Prazo (Figura 3).

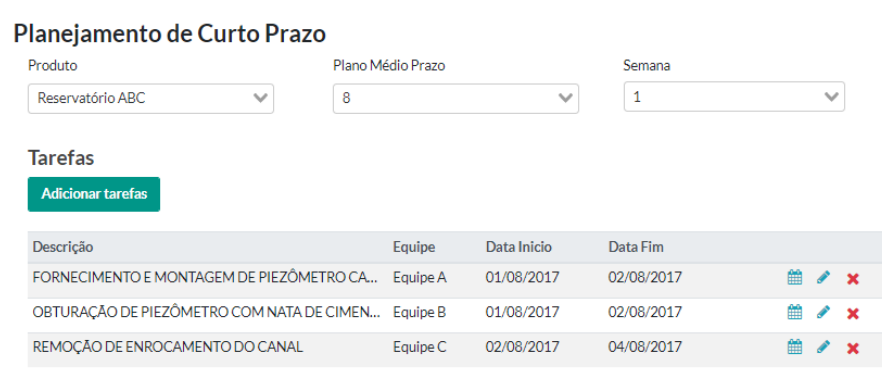

Figura 3. Exemplo de tela de Planejamento de Curto Prazo (weekly work planning).

A partir das telas das Figuras 3 e 4, é possível realizar o planejamento individual das tarefas e observar os indicadores de desempenho da tarefa em questão como as ilustradas nas Figuras 5 e 6 . 
Descrição: OBTURAÇĀO DE PIEZÔMETRO COM NATA DE CIMENTO (2) 02/08/2017 Equipe: Equipe B

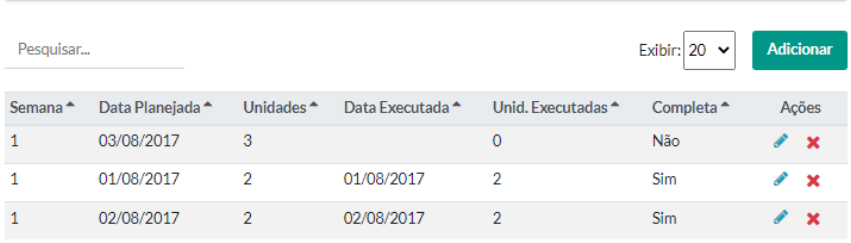

Figura 4. Exemplo de tela de Planejamento Individual da Tarefa.

Na Figura 5, nota-se que os dois indicadores de destaque são a Taxa de Produção Esperada e a Taxa de Produção Atual. A primeira é utilizada para indicar quanto de trabalho deve ser realizado para que seja possível alcançar o prazo planejado, sendo calculada através da razão entre o total de unidades planejadas (7 unidades) e o número de dias comprometidos (3 dias) para a execução da tarefa. Por isso, a Taxa de Produção Esperada é de 2,33 no exemplo. Já a Taxa de Produção Atual é calculada pela quantidade total de unidades executadas ( 4 unidades) pelo número de dias (3 dias). Por isso, é de 1,33. O PPC da tarefa também é calculado na proposta para indicar o percentual de conclusão da tarefa, conforme previsto no LPS. O PPC é calculado pela quantidade total de unidades executadas (4 unidades) pela quantidade total de unidades planejadas ( 7 unidades). $\mathrm{O}$ resultado é multiplicado por 100 para que o PPC seja apresentado em porcentagem. Por isso, o PPC tem valor 57, $14 \%$ no estudo de caso apresentado na Figura 5.

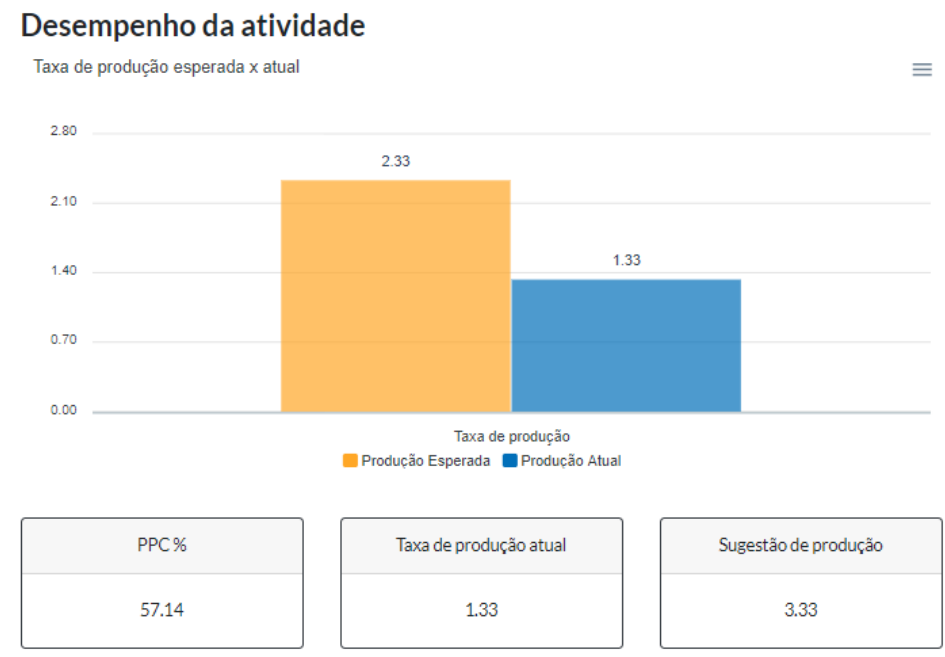

Figura 5. Exemplo de tela de indicadores da Taxa de Produção.

Já os indicadores apresentados na Figura 6 são criados a partir da aplicação das funções de singularidade (Etapa 3) no contexto dos registros do Planejamento Individual da Tarefa. O gráfico apresenta uma sugestão de melhoria na produção para que se consiga finalizar a tarefa dentro do que foi planejado.

\section{Conclusões}

O artigo apresentou uma proposta que serve para ajudar no Planejamento e Controle da Produção (PCP) considerando principalmente o aspecto relacionado aos atrasos. A pro- 


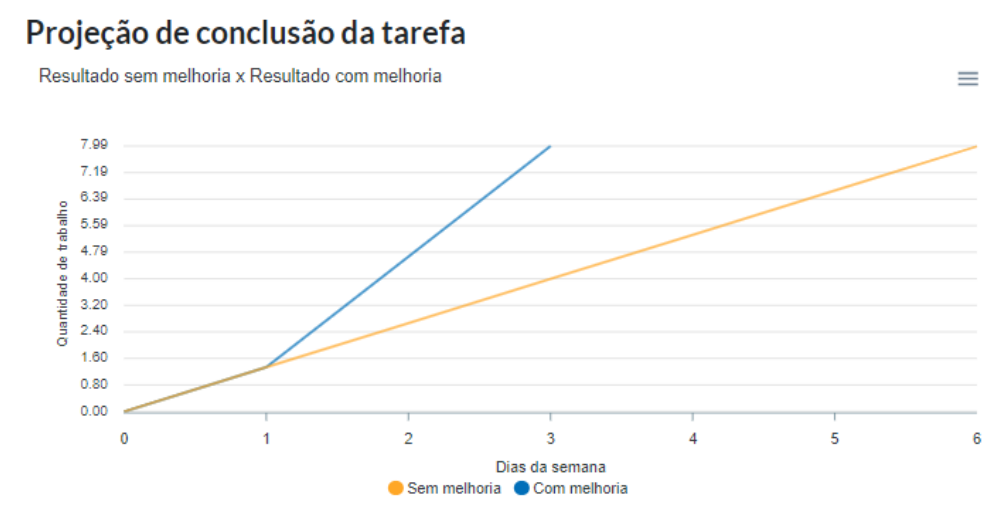

Figura 6. Exemplo de tela de Projeção de Conclusão de Tarefa.

posta foi integrada a um sistema real de gestão de construção civil. Um estudo de caso com dados reais foi apresentado mostrando a aplicação final da proposta no sistema e sua eficácia no contexto do objetivo proposto neste artigo. Assim, a contribuição deste trabalho para a IC está principalmente relacionada ao fato de que com a adoção de boas práticas e o uso de TICs é possível antecipar ações, bem como evitar atrasos e desperdício.

Como trabalhos futuros, pretende-se usar uma base de dados de projetos reais para aplicar algoritmos de aprendizagem de máquina que auxiliem na elaboração de cronogramas e orçamentos.

\section{Referências}

Abou Ibrahim, H., Hamzeh, F., Zankoul, E., Lindhard, S., and Rizk, L. (2019). Understanding the planner's role in lookahead construction planning. Production Planning and Control: The Management of Operations, 30(4).

Ballard, H. G. (2000). The Last Planner System of Production Control. PhD thesis, School of Civil Engineering Faculty of Engineering, University of Birmingham.

Batista, I., Feitosa, A., Douglas, L., Oliveira, R., Gurgel, M., Bezerra, A., Oliveira, C., and Braga, R. (2019). Bentham obras: Um sistema de gestão inteligente para a construção civil. In XXV Simpósio Brasileiro de Sistemas Multimídia e Web. SBC.

IBGE (2019). Pesquisa Anual da Indústria da Construção 2018. Intituto Brasileiro de Geografia e Estatística, Rio de Janeiro.

Lucko, G., Shehab, L., Ezzeddine, A., and Hamzeh, F. (2019). Singularity Functions for Early Warning Guidance in The Last Planner System. Lean Construction Journal $(L C J)$.

Moura, C. B. and Formoso, C. T. (2009). Análise quantitativa de indicadores de planejamento e controle da produção: impactos do Sistema Last Planner e fatores que afetam a sua eficácia. Ambiente Construído, 9(3).

Mubarak, S. A. (2015). Construction Project Scheduling and Control. Wiley, USA, 3rd edition.

Silva, P. H., Crippa, J., and Scheer, S. (2019). BIM 4D no planejamento de obras: detalhamento, benefícios e dificuldades. PARC Pesquisa em Arquitetura e Construção, 10 . 